\title{
Grenzen des laparoskopischen Operierens bei abdomineller Sepsis
}

\author{
Tobias Keck Ulrich Wellner Dietlind Tittelbach-Helmrich Dirk Bausch Konrad Karcz
}

Klinik für Chirurgie, Universitätsklinikum Schleswig-Holstein, Campus Lübeck, Deutschland

Schlüsselwörter

Laparoskopie · Sepsis · Abdomineller Fokus

\section{Zusammenfassung}

Hintergrund: Zahlreiche präklinische Daten weisen auf Vorteile des laparoskopischen Operierens auch bei abdomineller Sepsisquelle und septischem Patienten hin. Methode und Ergebnisse: Anhand derzeit verfügbarer Literatur betrachten wir die Möglichkeiten und Limitierungen laparoskopischen Operierens beim septischen Patienten mit abdominellem Fokus. Neben generellen Überlegungen werden im Speziellen das Vorgehen bei Appendizitis, Cholezystitis, perforiertem Ulkus, Sigmadivertikulitis und akuter Pankreatitis erörtert. Schlussfolgerungen: Erfahrene laparoskopische Chirurgen können die Sanierung der abdominellen Sepsisquelle bei Appendizitis, Cholezystitis, perforiertem Ulkus oder auch Sigmadivertikulitis sicher durchführen. Kombinationen aus interventionellen Techniken und minimal invasiven Operationen bieten insbesondere bei der perforierten Sigmadivertikulitis und bei infizierten Pankreasnekrosen innovative Ansätze, die derzeit in multizentrischen prospektiven Studien untersucht werden.

\section{Pathophysiologische Überlegungen und Gründe für das laparoskopische Operieren in der Sepsis}

In zahlreichen Teilgebieten der elektiven Chirurgie wurde durch den Einsatz laparoskopischer Operationen eine schnellere postoperative Genesung der Patienten mit konsekutiver kürzerer Verweildauer auf der Intensivstation und im Krankenhaus sowie mit weniger Schmerzen demonstriert. Septische Krankheitsbilder in der Viszeral-

\author{
Keywords \\ Laparoscopy $\cdot$ Sepsis $\cdot$ Abdominal focus
}

\section{Summary \\ Limitations of Laparoscopic Surgery for Abdominal Sepsis}

Background: Numerous preclinical data support the hypothesis of advantages of laparoscopic surgery for abdominal sepsis. Method and Results: We review the currently available literature and evaluate the possibilities and limitations of laparoscopic surgery in patients with an abdominal septic focus. General considerations are supported by a review of specific laparoscopic approaches for the patient with appendicitis, cholecystitis, perforated ulcer, sigmadiverticulitis, and acute pancreatitis. Conclusions: Experienced laparoscopic surgeons can safely perform surgery for abdominal sources of sepsis. Combinations of interventional and minimally invasive laparoscopic techniques are offering innovative approaches to sigmadiverticulitis and acute pancreatitis, which are currently evaluated in multicenter prospective trials.

chirurgie sind in der Regel mit einem intraabdominellen Fokus und den klassischen SIRS(systemisches inflammatorisches Response-Syndrom)-Kriterien Fieber oder Hypothermie, Tachykardie und Tachypnoe assoziiert. Inwieweit sich durch laparoskopisches Operieren in dieser Situation Vorteile für den Patienten ergeben und wo die Grenzen des laparoskopischen Operierens bei der abdominellen Sepsis liegen, soll im nachfolgenden Artikel dargestellt werden.

\section{KARGER \\ Fax +497614520714 \\ Information@Karger.com}

www.karger.com (c) 2013 S. Karger GmbH, Freiburg

1662-6664/13/0291-0028\$38.00/0

Accessible online at:

www.karger.com/vim
Univ.-Prof. Dr. med. Tobias Keck

Klinik für Chirurgie

Universitätsklinikum Schleswig-Holstein, Campus Lübeck

Ratzeburger Allee 160, 23538 Lübeck, Deutschland

tobias.keck@uksh.de 
Verschiedene tierexperimentelle und klinische Ergebnisse lassen den Einsatz des laparoskopischen Operierens beim septischen Patienten aus pathophysiologischer Sicht sinnvoll erscheinen. Die Anlage eines Pneumoperitoneums mit intraabdominellem $\mathrm{CO}_{2}$ in Tiermodellen konnte die Akute-PhaseAntwort des Organismus reduzieren [1]. Dies wurde in tierexperimentellen Sepsismodellen von einer geringeren Konzentration von peritonealen proinflammatorischen Zytokinen und Entzündungszellen als nach einer Laparotomie begleitet [2]. Bezüglich der bakteriellen Translokation, einem wichtigen pathophysiologischen Schritt im Progress der Peritonitis, sind die Daten weniger eindeutig. Hier konnte in verschiedenen tierexperimentellen Modellen sowohl eine reduzierte [3] als auch eine gesteigerte bakterielle Translokation $[4,5]$ durch den Einsatz eines Kapnoperitoneums gezeigt werden.

Neben der Beeinflussung kausaler pathophysiologischer Faktoren kann durch den Einsatz der Laparoskopie auch eine Immunmodulation erfolgen. Nguyen et al. [6] konnten für septische Schweine in einem experimentellen Großtiermodell zeigen, dass es durch den Einsatz der Laparoskopie im Vergleich zur Laparotomie zu einer geringeren Suppression der zellulären Immunität kommt. Inwieweit sich dieser Effekt auch auf die Immunkompetenz des Peritoneums übertragen lässt, ist unklar. Targarona et al. [7] konnten aufzeigen, dass der Einsatz der Laparoskopie zu einer besseren immunologischen Kontrolle des septischen Fokus führt, und dies trotz einer vorübergehenden Reduktion der Immunkompetenz. In zahlreichen weiteren Studien konnte über dies hinaus demonstriert werden, dass der Einsatz der Laparoskopie die Immunkompetenz des Organismus besser erhält [8, 9]. Des Weiteren konnte klinisch-experimentell dargelegt werden, dass der Einsatz eines Hochdruck-Pneumoperitoneums auch bei Vorliegen von intraabdominellen Infektionen und Peritonitis sicher ist $[10,11]$ und es nicht druckbedingt zur gesteigerten Einschwemmung von Bakterien kommt.

Ein weiterer wichtiger Faktor, der bei einer Laparoskopie in der Sepsis eine Rolle spielt, ist der Einsatz von $\mathrm{CO}_{2}$. Vor kurzem konnten Chatzimavroudis et al. [12] zeigen, dass durch den Einsatz von $\mathrm{CO}_{2}$ in einem druckkontrollierten Kapnoperitoneum experimentell das Überleben bei reduzierter Sepsisschwere verlängert werden konnte. Zahlreiche andere Berichte unterstützen die Hypothese, dass $\mathrm{CO}_{2}$ selbst in diesem Zusammenhang als bakteriostatischer Faktor wirken könnte $[13,14]$. Es gibt also präklinisch eine Vielzahl von Hinweisen, die den Einsatz der Laparoskopie auch im Rahmen der Behandlung des Fokus der abdominellen Sepsis unterstützen.

\section{Diagnostischer Einsatz der Laparoskopie beim septischen Patienten}

Neben der Intervention spielt die Laparoskopie auch eine zunehmende Rolle bei der Beurteilung von kritisch kranken Patienten auf der Intensivstation. Die möglichst rasche und früh-

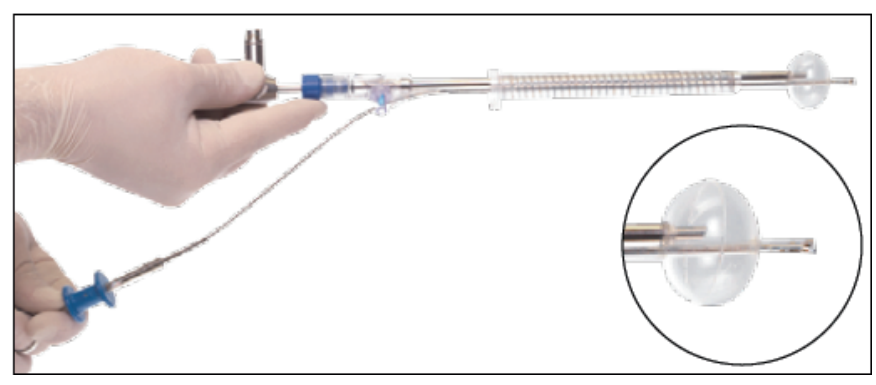

Abb. 1. Der Zwei-Kammern-Ballon (Firma Pajunk, Geisingen, Deutschland) für die Bedside-Laparoskopie garantiert, dass die Optik so nahe wie möglich an die zu untersuchende Stelle gelangt. Gleichzeitig sorgt er für den Abstand, der für eine gute Bildwiedergabe notwendig ist.

zeitige Diagnose des der Sepsis zugrunde liegenden abdominellen Fokus gestaltet sich beim intubierten und klinisch schlecht zu beurteilenden Patienten auf der Intensivstation zuweilen schwierig. Radiologische Schnittbildverfahren sind häufig aufgrund der sepsisbedingten Niereninsuffizienz nur mit geringer Aussagekraft und ohne Kontrastmittel durchzuführen. Intensivtransporte in die diagnostischen Einheiten sind personell aufwendig und häufig für den instabilen Patienten belastend. Ultraschalluntersuchungen sind untersucherabhängig und häufig in deren Ergebnissen nicht konklusiv [15, 16]. Ceribelli et al. [17] konnten in einer aktuellen Studie an 62 Intensivpatienten zeigen, dass die diagnostische Bedside-Laparoskopie sicher und effizient mit einer hohen diagnostischen Genauigkeit zur Fokussuche bei kritisch kranken, septischen Patienten durchgeführt werden kann. Peris et al. [18] zeigten, dass dieses Verfahren der reinen Peritoneallavage deutlich überlegen ist. So konnte durch die diagnostische Laparoskopie am Intensivbett bei 6 Peritoneallavage-negativen Patienten eine eitrige Peritonitis in deren Kollektiv nachgewiesen und somit die Indikation zur Operation gestellt werden. Das von uns im Rahmen der chirurgischen Intensivtherapie verwendete System mit Doppelballontechnik ermöglicht darüber hinaus die gasfreie Laparoskopie und ist gerade deshalb für den Einsatz auf der Intensivstation besonders geeignet (Abb. 1). Die diagnostische Aussagekraft beschränkt sich jedoch auf intraperitoneale Pathologien und ist bei retroperitonealen Prozessen eingeschränkt und unzureichend, wie auch andere Gruppen gezeigt haben [19].

\section{Spezielles Vorgehen bei bestimmten Krankheitsbildern}

Lokalisierte entzündliche, perforierte oder ischämische abdominelle Pathologien sind neben der initialen Diagnostik mittels Laparoskopie auch einer laparoskopischen Operation zugänglich.

\section{Perforierte Appendizitis}

Für die perforierte Appendizitis konnten Schietroma et al. [20] in einer Studie mit 147 konsekutiven Patienten zeigen, dass im 
Fall einer Peritonitis die offene Appendektomie zu einer gesteigerten Rate von Inflammation, Bakteriämie und Endotoxinämie führte als die laparoskopische Appendektomie. Eine transiente Reduktion der immunologischen Kompetenz bei den Patienten mit offener Appendektomie, repräsentiert durch ein Absinken des HLA(humanes Leukozyten-Antigen)-DR, ging in dieser Studie mit einer stärkeren Ausprägung der Sepsis einher. Wenn man die Gradierung der Komplikationen nach Clavien durchführt, dann kommt es nach einer Cochrane-Analyse aus dem Jahr 2004 bei den Patienten in der laparoskopischen Gruppe allerdings zu einer erhöhten Inzidenz intraabdomineller Abszesse (Komplikation Level 3 - Reintervention oder Reoperation) gegenüber einer erhöhten Rate an Level-1Komplikationen in der offenen Gruppe (vor allem Wundinfekte) [21]. Frühere Berichte [22] über eine erhöhte intraabdominelle Abszessrate bei Patienten mit perforierter Appendizitis konnten jedoch in neueren prospektiv randomisierten Studien, in denen sich im Gegenteil eine reduzierte Abszessrate bei erwachsenen und pädiatrischen Patienten in der laparoskopischen Gruppe zeigte, nicht mehr nachvollzogen werden [23, 24]. Im Fall einer perforierten laparoskopischen Appendizitis reduziert eine intraabdominelle Spülung nach derzeitiger Datenlage nicht die Inzidenz postoperativer Abszesse [25], wobei die derzeitige Evidenzlage hier bisher keine eindeutige Empfehlung erlaubt. Im Fall der Sepsis auf dem Boden einer perforierten Appendizitis besteht daher nach derzeitiger Evidenz keine Begrenzung des laparoskopischen Vorgehens.

\section{Perforierte Cholezystitis}

Die perforierte Cholezystitis als abdominelle Sepsisquelle birgt bei laparoskopischem Vorgehen ein erhöhtes Risiko iatrogener Gallengangsverletzungen [26]. Verlorene intraabdominelle Gallensteine verursachen bei freier Perforation oder akzidenteller perioperativer Verteilung im Bauchraum gemäß einer retrospektiven Studie [27] mehr postoperative Schmerzen, Ileus, mehr Infektionen an den Trokarstellen und konsekutiv einen verlängerten Krankenhausaufenthalt. Andererseits vermag die mangelnde Erfahrung mit der offenen Cholezystektomie bei vornehmlich laparoskopisch ausgebildeten Chirurgen außerhalb von hepatobiliären Zentren oftmals nicht die Sicherheit der Operation bei fortgeschrittener akuter Cholezystitis oder perforierter Cholezystitis zu erhöhen. Als Alternative in diesen Fällen wurde die laparoskopische partielle Cholezystektomie beschrieben. Dieses alternative Verfahren ist auch laparoskopisch unter Belassen eines längeren Infundibulum möglich. Die im Rahmen einer systematischen Übersichtsarbeit mit 625 Patienten [28] beschriebene höhere Rate an postoperativen Gallefisteln (10,6\%) mit diesem Verfahren war bei den meisten Patienten mit der Notwendigkeit einer postoperativen endoskopisch retrograden Cholangiopankreatikographie und Papillotomie oder Stenteinlage verbunden (7,5\%). Perkutane Interventionen waren bei $1,4 \%$ der Patienten nötig; in einem Fall kam es trotzdem zur Verletzung des Ductus choledochus und bei 2,2\% zu rekurrierenden Gallensteinen.
Für die perforierte Cholezystitis als abdominelle Sepsisquelle kann man zusammenfassen, dass je nach Erfahrung des Operateurs auch ein laparoskopisches Vorgehen - gegebenenfalls unter Verwendung der Technik der laparoskopischen partiellen Cholezystektomie - keine Limitierung darstellt. Je nach Erfahrung des Operateurs und intraoperativer Situation sollte jedoch zur Vermeidung einer Verletzung des Ductus choledochus und/oder Ductus hepaticus die Indikation zur Konversion und konventionellen Operation großzügig gestellt werden.

\section{Magenperforation/Duodenalperforation}

Liegt der abdominellen Sepsis eine Magen- oder Duodenalperforation zugrunde, so sind laparoskopische Techniken auch in der Notfallsituation anwendbar, wenn die logistischen Voraussetzungen gegeben sind und das Team in der laparoskopischen Chirurgie erfahren ist. In einer prospektiv randomisierten Studie [10] mit Beschränkung auf laparoskopisch erfahrene Chirurgen konnte gezeigt werden, dass die laparoskopische Operation im Vergleich zur offenen Operation signifikant länger dauerte und in der laparoskopischen Gruppe trotz der vorbestehenden Erfahrung der Chirurgen eine hohe Konversionsrate zum offenen Vorgehen bestand $(11 / 48,23 \%)$. Durch das laparoskopische Vorgehen konnte in dieser Studie keine Verkürzung des Kostaufbaus, der Krankenhausverweildauer, der Schmerzen, der Morbidität, der Komplikationsrate oder der Mortalität gezeigt werden. Unter Berücksichtigung von 96 Artikeln publizierten Lunevicius und Morkevicius [29] im Jahr 2005 eine Übersichtsarbeit zum laparoskopischen Management des Duodenalulkus. Auch wenn durch die Laparoskopie die Perforationsstelle in der Regel gut zu identifizieren ist, so ist die Unfähigkeit, dies zu tun, doch der häufigste Grund für eine Konversion. In einer Metaanalyse von Lau [30] aus dem Jahr 2004 wurden folgende Vorteile der laparoskopischen Operation des perforierten Duodenalulkus gesehen: geringerer perioperativer Schmerzmittelverbrauch sowie geringere Wundinfektionsrate und Mortalität. Diese Vorteile waren jedoch mit einer höheren Reoperationsrate verbunden. Die in den älteren Studien berichteten längeren Operationszeiten durch das laparoskopische Vorgehen konnten in einer prospektiv randomisierten Studie von Siu et al. [31] nicht nachvollzogen werden. Hier waren in spezialisierten Teams in der laparoskopischen Gruppe sogar kürzere Operationszeiten berichtet worden.

Zusammenfassend sind die Vorteile des laparoskopischen Vorgehens für das Management perforierter Magen- und Duodenalulzera nicht eindeutig nachvollziehbar. Derzeit ist davon auszugehen, dass die Operation in den meisten Fällen länger dauert und mit einer höheren Rate an Major-Komplikationen (Revisionsoperationen) verbunden ist. Diese beiden Faktoren sollten beim septischen Patienten vermieden werden. Daher sollte in dieser Konstellation nur ein überlegter Einsatz der Laparoskopie in erfahrenen Händen erfolgen, da die Grenzen des Nutzens des laparokopischen Vorgehens hier erreicht werden. 
Abb. 2. Die retroperitoneoskopische Nekrosektomie bei der nekrotisierenden Pankreatitis ist sicher und einfach durchführbar und ermöglicht die Kompartimentierung des Abdomens bei Möglichkeit der vollständigen

Nekrosektomie.

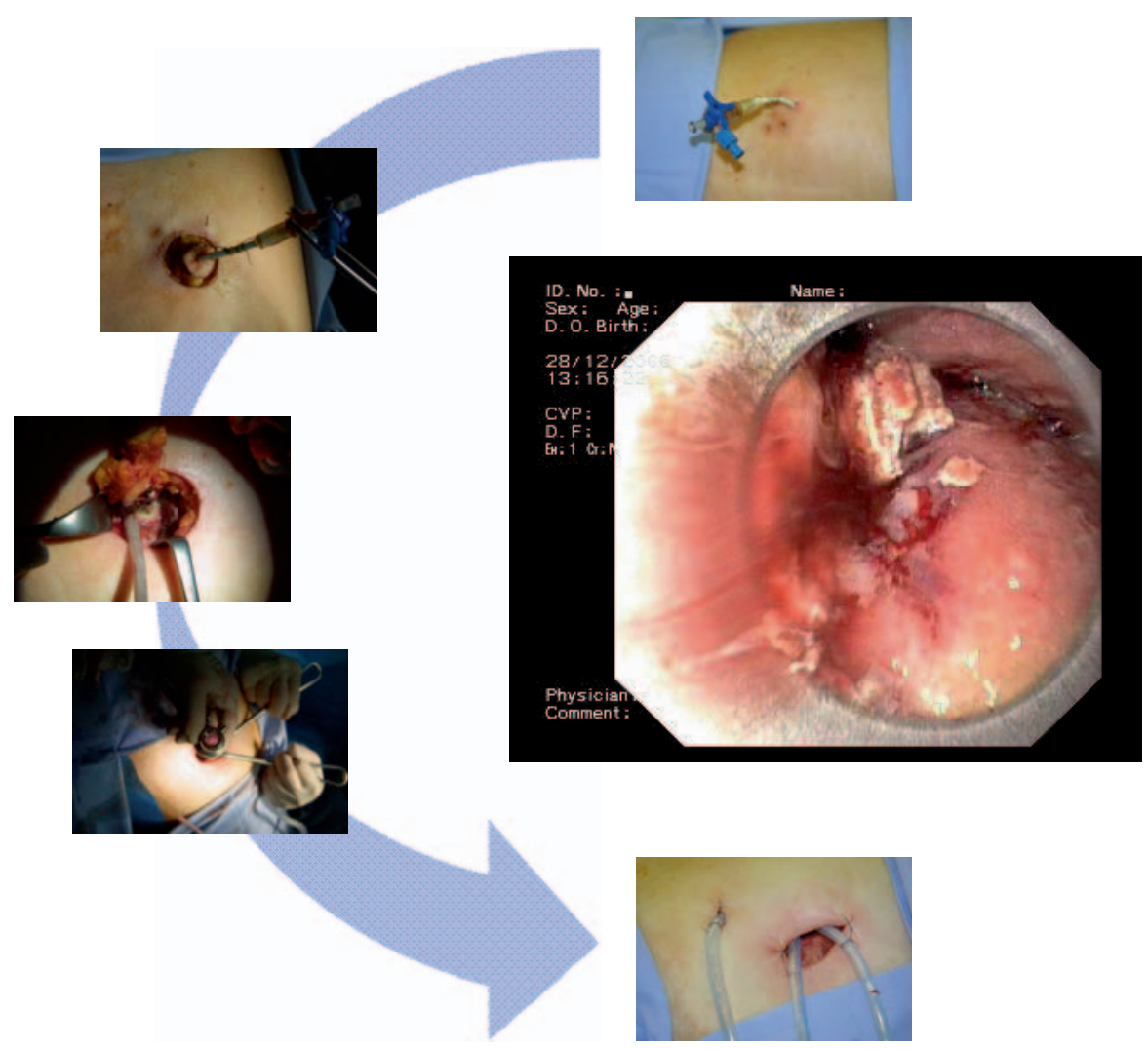

\section{Sigmaperforation}

Die freie Sigmaperforation mit kotiger Peritonitis als Quelle der abdominellen Sepsis stellt eine lebensbedrohliche Notfallindikation dar. Das Konzept der - der Kriegsmedizin entstammenden - Damage Control Surgery mit einer Minimierung des Eingriffs im septischen Zustand sieht auch in den gängigen Leitlinien zur kolorektalen Chirurgie die mehrzeitigen Operationen mit primärer Sigmaresektion und Rektumblindverschluss sowie Anlage eines endständigen Deszendostoma als Therapie der Wahl für Patienten mit generalisierter Peritonitis bei Sigmaperforation vor [32]. Dieses Konzept wird derzeit aufgrund zahlreicher Gründe hinterfragt. Die skizzierte Hartmann-Prozedur hat eine hohe perioperative Morbidität um 40\% und eine Mortalität von bis zu 20\% [33, 34]; des Weiteren besteht eine sehr geringe Rate an Kontinuitätswiederherstellung von unter $40 \%$. Liang et al. [35] haben in einer erst kürzlich publizierten Arbeit von ihren Erfahrungen mit zwei laparoskopischen Behandlungsstrategien - laparoskopische Lavage und Drainage sowie laparoskopische Hartmann-Operation - für die perforierte Sigmadivertikulitis berichtet. Mit der laparoskopischen Lavage und Drainage bei 47/88 Patienten berichten die Autoren erwartungsgemäß über kürzere Operationszeiten und Blutverlust, während nur 6,4\% der Patienten in dieser Gruppe aufgrund von septischem Progress akut mit einer Diskontinuitätsresektion operiert werden mussten. Weniger als die Hälfte der Patienten in der Gruppe mit Lavage und Drainage (21/47) mussten letztlich sekundär operiert werden. Somit befinden sich derzeit Verfahren in der
Entwicklung, die das Management des septischen Patienten mit dem Fokus der perforierten Sigmadivertikulitis auch durch laparoskopische Techniken ermöglichen. Die Ergebnisse des Scandinavian Diverticulitis Trial (SCANDIV, NCT01047462, www.clinicaltrials.gov) und des LADIES Trial (NCT01317485, www.clinicaltrials.gov) werden die derzeitigen Behandlungsregime der Sigmadivertikulitis mit einer gröBeren Bedeutung der Laparoskopie auch bei der Perforation beeinflussen.

\section{Nekrotisierende Pankreatitis}

Retroperitoneale Prozesse wie die nekrotisierende Pankreatitis sind durch transperitoneale Laparoskopie angehbar, doch widerspricht der transperitoneale Zugang zu Nekrosen des Pankreas der Vorstellung der Kompartimentierung und Abgrenzung des Fokus, was durch einen primär retroperitonealen Zugang oder einen transgastralen Zugang besser erreicht werden kann. Eine abdominelle Sepsis durch Nekrosen des Pankreas sollte, wenn möglich, nicht innerhalb der ersten 4-6 Wochen nach Krankheitsbeginn operiert werden. Danach steht entsprechend des Step-Up-Vorgehens ein breites Spektrum und Armamentarium an interventionellen Techniken zur Verfügung. Durch die Inklusion von interventionellen Techniken und retroperitonealer Drainage war es in dem prospektiv randomisierten PANTER Trial [36] möglich, durch den sequenziellen Einsatz von interventionellen und minimal invasiven retroperitoneoskopischen Techniken eine geringere Inzidenz von Organversagen (12 vs. 40\%) sowie von Hernien 
im Vergleich zum konventionellen, offenen Vorgehen der Nekrosektomie zu demonstrieren (7 vs. 24\%). Die Mortalität wurde durch dieses sequenzielle Vorgehen nicht reduziert (19 vs. $16 \%)$. Babu et al. [37] evaluierten in einer erst kürzlich erschienenen Studie dieses sequenzielle Vorgehen für 70 Patienten mit nekrotisierender Pankreatitis. 55\% der Patienten konnten dabei lediglich mittels intensivmedizinischer Behandlung und interventionellen Drainagen behandelt werden. Wir konnten zeigen, dass der Einsatz der minimal invasiven retroperitoneoskopischen Nekrosektomie (Abb. 2) bei Patienten, die in einem Step-Up-Vorgehen aufgrund septischer Konstellation operiert werden müssen, in $57 \%$ primär erfolgreich war [38]. Für retroperitoneale Sepsisquellen wie z.B. infizierte Pankreasnekrosen sind sequenzielle Interventionsund minimal invasive Operationsverfahren sichere Alternativen zum klassischen, offen chirurgischen Vorgehen. Die Verfahren sind effizient und mit einem geringeren Risiko der Narbenbildung verbunden.

\section{Schlussfolgerungen für die Klinik: Grenzen des laparo- skopischen Operierens bei abdomineller Sepsis}

Präklinische Daten lassen vermuten, dass der Einsatz der Laparoskopie und eines Kapnoperitoneums immunmodulatorische protektive Effekte beim septischen Patienten haben könnten. Lokalisierte Entzündungsprozesse im Abdomen und Retroperitoneum können gut minimal invasiv beherrscht werden. Bei diffusen Entzündungen und diffuser Peritonitis scheinen das interventionsbedürftige Abszessrisiko und die schwerwiegenden Komplikationen (Clavien III) durch das laparoskopische Vorgehen höher zu sein als der Nutzen des minimal invasiven Zugangsweges. Für spezifische Krankheitsbilder muss aufgrund der hohen Mortalität der abdominellen Sepsis insbesondere bei laparoskopisch weniger erfahrenen Chirurgen die schnelle und sichere Sanierung des abdominellen Fokus immer im Vordergrund - d.h. vor Fragen der Minimalisierung des Zugangsweges - stehen. Risikofaktoren für ein Scheitern der laparoskopischen Operation sind eine Dauer der Perforation $>24$ h, septischer Schock, zugrunde liegende Komorbidität, ASA III-IV und ein APACHE-II-Score >5 Punkte [29]. Erfahrene laparoskopische Chirurgen können die Sanierung der abdominellen Sepsisquelle bei perforierter Appendizitis, Cholezystitis, Ulkus oder auch Sigmadivertikulitis sicher durchführen. Kombinationen aus interventionellen Techniken und minimal invasiven Operationen bieten insbesondere bei der perforierten Sigmadivertikulitis und bei infizierten Pankreasnekrosen innovative Ansätze, die derzeit in multizentrischen prospektiven Studien untersucht werden.

\section{Disclosure Statement}

TK, UW, DT-H und DB haben keine Interessenkonflikte. KK ist Medical Advisor für Pajunk und Covidien.

\section{Literatur}

1 Hanly EJ, Mendoza-Sagaon M, Murata K, Hardacre JM, De Maio A, Talamini MA: $\mathrm{CO}_{2}$ pneumoperitoneum modifies the inflammatory response to sepsis. Ann Surg 2003;237:343-350.

2 Matsumoto T, Dolgor B, Ninomiya K, Bandoh T, Yoshida T, Kitano S: Effect of $\mathrm{CO}_{2}$ pneumoperitoneum on the systemic and peritoneal cytokine response in a LPS-induced sepsis model. Eur Surg Res 2001;33:71-76.

3 Karantonis F-F, Nikiteas N, Perrea D, Vlachou A, Giamarellos-Bourboulis EJ, Tsigris C, Kostakis A Evaluation of the effects of laparotomy and laparoscopy on the immune system in intra-abdominal sepsis - a review. J Invest Surg 2008;21:330-339.

$\checkmark 4$ Ozmen MM, Cöl C, Aksoy AM, Tekeli FA, Berberoglu M: Effect of $\mathrm{CO}_{2}$ insufflation on bacteremia and bacterial translocation in an animal model of peritonitis. Surg Endosc 1999;13:801-803.

$\checkmark 5$ Evasovich MR, Clark TC, Horattas MC, Holda S, Treen L: Does pneumoperitoneum during laparoscopy increase bacterial translocation? Surg Endosc 1996;10:1176-1179.

6 Nguyen NT, Luketich JD, Schatz S, Tran Q, Ho HS, Schauer PR: Effect of open and laparoscopic surgery on cellular immunity in a swine model. Surg Laparosc Endosc Percutan Tech 1999;9:176-180.

7 Targarona EM, Rodríguez M, Camacho M, Balagué C, Gich I, Vila L, Trias M: Immediate peritoneal response to bacterial contamination during laparoscopic surgery. Surg Endosc 2006;20:316-321.
8 Klava A, Windsor A, Boylston AW, Reynolds JV, Ramsden CW, Guillou PJ: Monocyte activation after open and laparoscopic surgery. Br J Surg 1997:84:1152-1156.

$\checkmark 9$ Redmond HP, Hofmann K, Shou J, Leon P, Kelly CJ, Daly JM: Effects of laparotomy on systemic macrophage function. Surgery 1992;111:647-655.

10 Lau WY, Leung KL, Kwong KH, Davey IC, Robertson C, Dawson JJ, Chung SC, Li AK: A randomized study comparing laparoscopic versus open repair of perforated peptic ulcer using suture or sutureless technique. Ann Surg 1996;224:131-138.

11 Navez B, Tassetti V, Scohy JJ, Mutter D, Guiot P, Evrard S, Marescaux J: Laparoscopic management of acute peritonitis. Br J Surg 1998;85:32-36.

12 Chatzimavroudis G, Pavlidis TE, Koutelidakis I, Giamarrelos-Bourboulis EJ, Atmatzidis S, Kontopoulou K, Marakis G, Atmatzidis $\mathrm{K}: \mathrm{CO}_{2}$ pneumoperitoneum prolongs survival in an animal model of peritonitis compared to laparotomy. J Surg Res 2009;152:69-75.

13 Evrard S, Falkenrodt A, Park A, Tassetti V, Mutter D, Marescaux J: Influence of $\mathrm{CO}_{2}$ pneumoperitoneum on systemic and peritoneal cell-mediated immunity. World J Surg 1997;21:353-356; discussion 357.

14 Taffinder NJ, Cruaud P, Catheline JM, Bron M, Champault G: Bacterial contamination of pneumoperitoneum gas in peritonitis and controls: a prospective laparoscopic study. Acta Chir Belg 1997;97:215-216.
15 Wang H-P, Chen S-C: Upper abdominal ultrasound in the critically ill. Crit Care Med 2007;35: S208-215.

16 Crandall M, West MA: Evaluation of the abdomen in the critically ill patient: opening the black box. Curr Opin Crit Care 2006;12:333-339.

17 Ceribelli C, Adami EA, Mattia S, Benini B: Bedside diagnostic laparoscopy for critically ill patients: a retrospective study of 62 patients. Surg Endosc 2012;26:3612-3615.

18 Peris A, Matano S, Manca G, Zagli G, Bonizzoli M, Cianchi G, Pasquini A, Batacchi S, Di Filippo A, Anichini V, Nicoletti P, Benemei S, Geppetti P: Bedside diagnostic laparoscopy to diagnose intraabdominal pathology in the intensive care unit. Crit Care 2009;13:R25.

19 Stefanidis D, Richardson WS, Chang L, Earle DB, Fanelli RD: The role of diagnostic laparoscopy for acute abdominal conditions: an evidence-based review. Surg Endosc 2009;23:16-23.

20 Schietroma M, Piccione F, Carlei F, Clementi M, Bianchi Z, de Vita F, Amicucci G: Peritonitis from perforated appendicitis: stress response after laparoscopic or open treatment. Am Surg 2012;78:582-590.

21 Sauerland S, Lefering R, Neugebauer EAM: Laparoscopic versus open surgery for suspected appendicitis. Cochrane Database Syst Rev 2004;4: CD001546.

22 Lippert H, Koch A, Marusch F, Wolff S, Gastinger I: Offene versus laparoskopische Appendektomie. Chirurg 2002;73:791-798 
23 Wei H-B, Huang J-L, Zheng Z-H, Wei B, Zheng F, Qiu W-S, Guo W-P, Chen T-F, Wang T-B: Laparoscopic versus open appendectomy: a prospective randomized comparison. Surg Endosc 2010;24 266-269.

24 Wang X, Zhang W, Yang X, Shao J, Zhou X, Yuan $\mathrm{J}$ : Complicated appendicitis in children: is laparoscopic appendectomy appropriate? A comparative study with the open appendectomy - our experience. J Pediatr Surg 2009;44:1924-1927.

25 Moore CB, Smith RS, Herbertson R, Toevs C: Does use of intraoperative irrigation with open or laparoscopic appendectomy reduce post-operative intra-abdominal abscess? Am Surg 2011;77:78-80.

26 Kholdebarin R, Boetto J, Harnish JL, Urbach DR: Risk factors for bile duct injury during laparoscopic cholecystectomy: a case-control study. Surg Innov 2008;15:114-119.

-27 Suh SW, Park JM, Lee SE, Choi YS: Accidental gallbladder perforation during laparoscopic cholecystectomy: does it have an effect on the clinical outcomes? J Laparoendosc Adv Surg Tech A 2012; 22:40-45.
28 Henneman D, da Costa DW, Vrouenraets BC, van Wagensveld BA, Lagarde SM: Laparoscopic partial cholecystectomy for the difficult gallbladder: a systematic review. Surg Endosc 2012;DOI: 10.1007/ s00464-012-2458-2.

29 Lunevicius R, Morkevicius M: Management strategies, early results, benefits, and risk factors of laparoscopic repair of perforated peptic ulcer. World J Surg 2005;29:1299-1310.

30 Lau H: Laparoscopic repair of perforated peptic ulcer: a meta-analysis. Surg Endosc 2004;18:10131021.

31 Siu WT, Leong HT, Law BKB, Chau CH, Li ACN, Fung KH, Tai YP, Li MKW: Laparoscopic repair for perforated peptic ulcer: a randomized controlled trial. Ann Surg 2002;235:313-319.

32 Oliver G, Lowry A, Vernava A, Hicks T, Burnstein M, Denstman F, Fazio V, Kerner B, Moore R, Peters W, Ross T, Senatore P, Simmang C, Wexner S, Wong WD: Practice parameters for antibiotic prophylaxis supporting documentation. The Standards Task Force. The American Society of Colon and Rectal Surgeons. Dis Colon Rectum 2000;43:1194-1200.

33 Seah DW, Ibrahim S, Tay KH: Hartmann procedure: is it still relevant today? ANZ J Surg 2005; 75:436-440.
34 Salem L, Anaya DA, Roberts KE, Flum DR: Hartmann's colectomy and reversal in diverticulitis: a population-level assessment. Dis Colon Rectum 2005;48:988-995.

35 Liang S, Russek K, Franklin ME Jr: Damage control strategy for the management of perforated diverticulitis with generalized peritonitis: laparoscopic lavage and drainage vs. laparoscopic Hartmann's procedure. Surg Endosc 2012;26:2835-2842.

36 van Santvoort HC, Besselink MG, Bakker OJ, et al.: A step-up approach or open necrosectomy for necrotizing pancreatitis. N Engl J Med 2010;362: 1491-1502.

37 Babu RY, Gupta R, Kang M, Bhasin DK, Rana SS, Singh R: Predictors of surgery in patients with severe acute pancreatitis managed by the step-up approach. Ann Surg 2012;DOI: 10.1097/SLA.0b013e318269d25d.

38 Bausch D, Wellner U, Kahl S, Kuesters S, RichterSchrag H-J, Utzolino S, Hopt UT, Keck T, Fischer A: Minimally invasive operations for acute necrotizing pancreatitis: comparison of minimally invasive retroperitoneal necrosectomy with endoscopic transgastric necrosectomy. Surgery 2012;152:S128-134. 